\title{
Research on the Continuous Use Intention of Government Portals Based on Grounded Theory
}

\author{
Zhiwei Tang ${ }^{1}$, Jing $\operatorname{Tan}^{1}$, Zepeng Gong ${ }^{1, *}$, Xiao $\operatorname{Han}^{2}$ and Seth kwame sakyi Arthur ${ }^{1}$ \\ ${ }^{1}$ School of Political Science and Public Administration, University of Electronic Science and Technology of China, Chengdu, P. R. \\ China, 611731 \\ ${ }^{2}$ School of International and Public Affairs, Shanghai Jiaotong University, Shanghai, P. R. China, 200030 \\ ${ }^{*}$ Corresponding author
}

\begin{abstract}
Since the first investigation of government websites in our country in 2015, problems such as low utilization rate and poor content construction have appeared. To solve the realistic problems and make up on the previous studies, this paper, based on grounded theory, discusses the factors influencing public continuous use of government websites by analyzing data from 56 interviewees, and establishes a theoretical model for influential factors of the public continuous use of government websites through open coding, axial coding, selective coding and other procedures. This research finds the need-perception fit as the direct influential factor of continuous use of government websites. Service operation, government's ideas, users' characteristics and external environment of government websites indirectly influence the continuous use intention.
\end{abstract}

Keywords-grounded theory; government websites; continuous intention; influential factors

\section{INTRODUCTION}

E-government is a channel in which government departments use various information technologies in releasing information to the public, online service supply, online feedback and interactive communication ${ }^{[1]}$. Its purpose is to improve government's efficiency of handling affairs and providing better services to the public. To achieve this purpose, governments around the world invest lots of resources on constructing government websites to release government information, supply service and enhance interactions with the public $^{[2]}$. E-government in China started in the 1980s. After 20 years' development, governments at all levels have established E-government platforms. The number of Chinese government websites was 84,094 by the end of November, $2015^{[3]}$, representing $2.36 \%$ of the $3,570,000$ Chinese websites. The number of Chinese Internet users reached 668,000,000, representing half of the total Chinese population ${ }^{[4]}$. Nonetheless, there are many citizens who have never used government websites or know little about their functions. The survey report on E-government construction in Fujian Province in 2011 showed that $81 \%$ of the respondents had never visited local government websites; $93.4 \%$ of companies preferred traditional channels when they needed to communicate with government departments, only $6.6 \%$ of the respondents chose online channels ${ }^{[5]}$. Ma Liang (2014) made an investigation on the citizens from 36 major cities in China, finding that nearly half of the residents had never logged into government websites ${ }^{[6]}$. The low utilization of government websites of China is also a dilemma encountered by other countries in their construction of E-government.

As an important part of E-government construction, government website is an important window for government to provide services for citizens, and an important platform for governments to communicate with the public. The ability of service supply of government website directly influences the fulfillment of government function.

\section{LitERATURE REVIEW}

The ultimate goal of Information System is its continuous use, so users' continuous use is a key to the success of Information System. Considering the fact that only a few of studies both at home and abroad concentrate on the continuous use of government website, which is also an information system, this paper reviewed literature about users' continuous use of Information System to avoid omission. The theory of Behavioral Intention is widely used in the field of Egovernment, such as TAM, TPB, TTF, UTAUT. These models demonstrated the influential factors of users' adoption by empirical studies. However, these models cannot effectively explain users' continuous use intention for they focus on the initial adoption of Information System. Although these information technology adoption theory models have been modified and developed in the perspective of communication and social psychology, their explanations on the continuous use behavior are still weak. In order to explore the influential factors of users' continuous use of information system, Bhattacherjee (2001) breaking through the research framework of Technology Acceptance theory, constructed a new theory model for continuous use of information system by introducing the new expectation confirmation theory and referring to theoretical framework and different variables (including satisfaction, users' habits and so on ), and testified the model through empirical studies, making great theoretical contribution to the research on users' continuous use behavior $^{[7]}$.

Since then, the academics have maintained a keen attention on continuous use intention and put forward a lot of constructive opinions. Such as Guopeng Yin (2014) found that using experience, entertainment, social interaction, satisfaction and habits will affect users' continuous use of social networking sites ${ }^{[8]}$. Deliang Wang, Lingling $\mathrm{Xu}$, Hock Chuan Chana (2015), basing on the perspective of computer self- 
efficacy, integrated the social cognitive theory with thinkingemotion balancing model, constructed a model of continuous use of social media, and found that cognition, preference, and special computer self-efficacy are the main factors affecting the continuous use intention ${ }^{[9]}$.

The studies mentioned above almost confirmed all possible influential factors of continuous use, and made great effects on the study of continuous use. However, the theoretical variables of these empirical researches were derived from researches of business information system, and neglected the real environment of governmental information system. The goal of business information system is totally different from that of the governmental information system; these models lack credibility when used to explain the continuous use of governmental information system. Empirical researches lead to the simplification, fragmentation, lack of macro and overall research perspectives, and hinder the discovery of new theoretical factors. Although, a few scholars have tried to study on the problem from an overall perspective or applying qualitative research method, they failed to appropriately explain the influential mechanism of users' continuous use intention of government websites and cannot put forward operational advice. Therefore, the scholars in this field should continue to ask: what are the influential mechanisms of continuous use of government websites? How to improve the public's continuous use of government websites? To solve these questions, this paper plans to use an inductive approach to conduct a systematic research, in order to break the framework of empirical research, look for new theoretical factors and propose better explanations and operational suggestions.

\section{RESEARCH DESIGN AND MODEL CONSTRUCTION}

\section{A. Research Methods and Data Collection}

The Grounded Theory is an integrated research method aiming at forming a new theory to explain and study on certain related behavioral modes, rather than verifying the existing theories $^{[10]}$. As a rigorous and complete qualitative research method, the Construction Grounded Theory has been widely accepted by scholars, and it has been successfully applied to the field of information systems. Therefore, this paper adopts the Construction Grounded Theory as research method to study on the public continuous use intention of government's websites. The research process is as follows (see Figure 1).

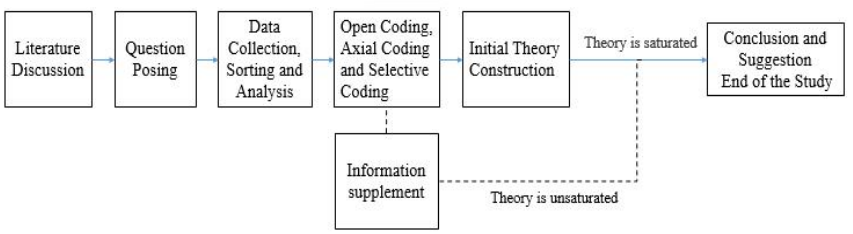

FIGURE I. FLOW CHART OF GROUNDED THEORY

Note: The information is from literature ${ }^{[11]}$.

The questionnaire was designed based on the theme of the study. After consulting 8 experts and scholars in the field of Egovernment, and having a completely open discussion with two postgraduate students who major in Public Administration, an open interview outline was finalized. This paper obtained research data through one to one in depth interviews with respondents. Each interview lasted about 35 minutes, and after the interview ended, coding was processed immediately. Once the study reached theoretical saturation, the data collection ended. The selection of the respondents regarded the realization of theoretical saturation as principle, applied the theoretical sampling method, followed the requirement of the category generation and theory development, and combined with the research theme of this paper. A total of 56 respondents were interviewed, and all of them had used government websites or participated in the construction of government websites. A large quantity of data was obtained through 5 months' continuous interviews (from May 21, 2015 to November 5, 2015). This paper analyzed data systematically, and when no new category appearing, it comparatively analyzed the relevant data collected from literature review.

\section{B. Opening Coding}

Open Coding is a process of decomposition, validation, comparison, conceptualization, and data classification. "What kind of research does the data involve with?”, "What does the data represent?" and "which theoretical category does the data indicate?" Keeping these questions in mind, researchers generalized and categorized data according to its own natural status by staying close to data, making codes simple and precise, and analyzing all data word by word with open and careful attitude. In this paper, the respondents were numbered according to the interview sequence. FT01 represented the first respondent and so on. Finally, we get 31 categories.

\section{Axial Coding}

Axial coding is a process of comparing and analyzing relations of categories obtained through open coding that belong to different levels, types and have unclear relations. It classifies the categories, links them together and forms the main categories. In this paper, 13 main categories were produced by axial coding: B1 government concepts; B2 service operation of government websites; B3 users' characteristics; B4 psychological perceptions; B5 users' needs; B6 quality of service; B7 quality of information; B8 quality of system; B9 alternative resources; B10 subjective norm; B11 fundamental environment; B12 legal environment; B13 economic environment. For Grounded Theory can code not only interview data but also the previous literature, this paper reviewed literature in this field and found that social culture was associated with people's value, and could affect people's behavioral intention. So this paper classified social value as the $14^{\text {th }}$ main category, B14.

\section{Selective Coding}

Selective Coding is a process based on axial coding. This process systematically analyzes and debugs the main categories obtained from axial coding to get core categories that can generalize other related categories ${ }^{[12]}$. Based on the selective coding of 14 main categories, this paper classified "government concepts" and "users' characteristics" into a 
separate core category. Then we make "service operation of government websites", "quality of service", "quality of information" and "quality of system" belong to the core category "service operation of government websites", and make "psychological perceptions" and "users' needs" belong to the core category "need-perception fir", and make "alternative resources”, "subjective norm”, "infrastructure", "legal environment", "economic environment" and "social culture" belonged to the core category "external environment of government websites". The 5 core categories together influenced the public's continuous use intention.

\section{E. Model Construction and Saturation Test}

\section{1) Reliability and validity tests}

The reliability means that the results are achieved through complete and consistent methods and are credible. We randomly selected half processed data of one coder, and let another coder process it again. According to the categories obtained from the data, we calculated the category quantities that the two (2) coders agreed on and disagreed with, and got the reliability of $97.03 \%$. To test the validity, this paper adopted inspection of members. The researcher inspected the analyzed materials to confirm whether they were consistent with his ideas or experience. In this research, we have presented the analysis of interview data and coding results to the corresponding seven (7) respondents to check if the results were consistent with their ideas. The respondents put forward some comments and we did modifications accordingly out of respect for them.

\section{2) Model construction:}

The government website is a resource platform which provides information and service to the public. How to make full use of it? What factors influence the public's continuous use intention? This paper coded the interview data in three levels and presented five factors that influenced people's continuous use intention and their relations: (1) government concepts directly influenced the service operation of the government website; (2) the service operation of government website directly influenced users' need-perception fit; (3) users' need-perception fit directly influenced their continuous use intention of government websites. The external environment influenced government concepts, service operation of government websites and users' demandperception fit. Users' characteristics influenced both service operation of government websites and demand-perception fit. This paper therefore constructed an initial theoretical model of public continuous use intention toward government websites (see Figure 2).

\section{3) Theoretical saturation test}

Combining with predecessors' ideas and experience of theoretical saturation, researchers tested the theoretical saturation of this research with three questions: "what kind of comparison was made between data and categories?", "how did the comparison explain the categories?", and "were there any other directions? What new conceptual relations would have been produced if the answer was Yes?” After repeated comparison analysis of the interview data and literature review, we did not find any new concept relation. So the theoretical model of this paper is saturated.

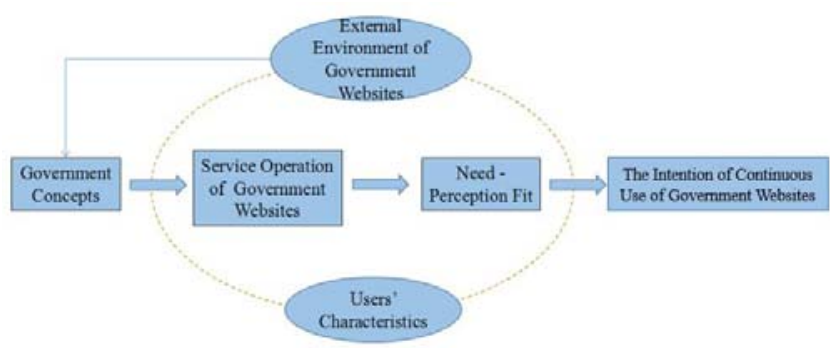

FIGURE II. THEORETICAL MODEL OF PUBLIC CONTINUOUS USE INTENTION OF GOVERNMENT WEBSITE

\section{RESEARCH RESUlts AND ANALysis}

The conceptual model shows that "government concepts", "service operation of government websites", "need perception fit", "users' characteristics" and "the external environment of government websites" together influenced public continuous use of government websites.

\section{A. Core Category "Government Concepts"}

Government is the constructor and servant of government websites. So the core category of "government concepts" is an influential factor of continuous use of government websites and it contains three sub-categories: the informationalized ideas of governments, governments' emphasis and the concept of service oriented government. The informationalized ideas of government refers to the thinking mode of informational service; governments' emphasis refers to the importance that government departments and leaders attach to government websites; the concept of service oriented government refers to the concept that government should serve the public as its basic tenet of website construction, operation and development. In the model, "government concepts" directly affect "service operation of its websites". Respondent nine (9) said "government leaders and staff get used to the traditional seals and seldom accept the electric seals. It will disturb the development of online work flows". The typical "path dependence" presents that government's informationalized ideas are still weak and the introduction of network technology does not break through the path of the system. Thus, the service operation of government websites is limited. In addition, the service attitude of government staff is affected by leaders, because the concept of constructing service oriented government is not deeply rooted among the public.

\section{B. Core Category "Service Operation of Government Websites"}

The core category "service operation of government websites" refers to the management and protection of service operation process and the design, development, maintenance and control of government websites, including 4 sub categories: service operation, service quality, information quality and system quality. The service operation provides supports to the service of government websites, such as setting up specialized function structures and inputting financial resources. Service quality, information quality and system 
quality are the construction content of government websites. Whether the websites consider and satisfy users' needs, and protect users' interests when offering service is the content of service quality. Information quality contains availability, reliability, effectiveness and entertainment of the service provided by the websites and system information covers the safety and stability of website systems, the compatibility of browsers and the response speed to users' operation. In the model, "service operation of government websites" has an impact on "need-perception fit". Respondent forty-nine (49) said "I once worked at a construction site. One of my workmates broke his leg broken. We didn't know which hospitals rendered better services. I had heard that information provided by was precise, so I immediately logged into the government website on my mobile phone and found the appropriate orthopedic hospital. For this experience, I am so satisfied and I will look for information on government websites". We found the direct reason of his continuous use intention was his satisfaction of the comprehensiveness, usefulness and convenience of government websites.

\section{Core Category "Need - Perception Fit"}

The core category "need-perception fit" indicates users' psychological perception of their satisfaction degree and service obtained after using government websites. It contains two subcategories: psychological perception and users' needs. Users' needs refers to the needs based on their own situation, and psychological perception refers to users' psychological status and feelings before, during and after they use government websites, including the sense of immediacy, using desire, perception of usefulness, perception of convenience, irreplaceability, perception of trust, perceived cost, satisfaction and so on.

The government website is an information system, and its continuous use entirely depends on users. Hence, the core category "need - perception fit" directly affects users' intention on continuous use of government websites. Users use government websites based on their psychological conditions and needs, and they will form certain psychological perceptions during the process: whether the website's service matches their needs; their psychological feeling when they use the website to meet their needs; the satisfaction degree of using and so on. Respondent twenty-two (22) said "I use the Internet only for movies. I've never thought about using the Internet to deal with affairs related to the government. There was a time, my parents needed to apply for the preferential cards for the aged. My friend suggested that I search information from the government website. I tried to use the website and it was easy for me to get the detailed information. Since then, I began to use government website".

\section{Core Category "Users' Characteristics"}

"Users' characteristics” affect both "service operation of government websites" and "need - perception fit". It refers to specific users of government websites, their basic conditions, habits, and abilities and includes 4 subcategories: user group, demographic variables, habits and individual abilities. Government's service operation of websites should recognize its target users, design the web pages and functions, and provide information and services according to users' situations. Respondent twenty-seven (27) said "I think the construction of government websites ignores the public's opinions. They never ask what we need. If the service is close to life and is related to our own benefit, we will use the websites frequently". And we found that the "users' characteristics" influence the "need - perception fit" from the interview.

\section{E. Core Category "External Environment of Government Websites"}

The core category "external environment of government websites" refers to the collection of things surrounding government websites and the influence it exerts on websites. This category contains six subcategories: alternative resources, subjective norm, infrastructure, economic environment and legal environment. Fang Wang (2014) thought overlapping, complementary, competitive and replaceable relations exist between government portals and other information channels for resources ${ }^{[13]}$. The government release, government microblog, television, newspaper, on-site affairs handling and other alternative methods have negative impacts on the public's irreplaceability perception of government websites. In this paper, subjective norm refers to the influence of other people and groups and it affects users' psychological perception and needs. Infrastructure refers to the objective hardware conditions that influence the construction, popularization and use of government websites. Legal environment influences government concepts, website construction, service mechanism and users' security perception. The development of the economy is conducive to the construction of infrastructure and government's support of manpower and material resources. Social culture influences government concept, service operation of government websites, users' needs and psychological perception. Therefore, the core category "external environment of government websites" has an influence on "government concept", "service operation of government websites” and "need-perception fit”.

\section{CONCLUSION AND PROSPECTS}

With the guide of Grounded Theory, and through the analysis of interview data, we got the five core categories: "government concepts", "service operation of government websites”, "need - perception fit”, "users' characteristics” and "external environment of government websites". Besides, this paper constructed the theoretical model of the influential factors of public's continuous use intention of government websites. Among them, "government concepts" and "external environment of government websites" are new influential factors. The "need - perception fit" is the direct cause of the continuous use of government websites and the other four core categories are the indirect causes. Above all, this paper puts forward four suggestions.

(1) Meet the diversified needs of the public, innovate services, and achieve precise service of government websites

Firstly, the construction of government websites should insist on the basic principle of serving the public, and meet diversified requirements of different user groups. Secondly, improve data mining and analyzing abilities of government 
websites to understand public demands more precisely. In addition, government websites should rise to the challenge of new medias, set up service brand and strengthen the irreplaceability and competitiveness of government websites.

(2) Improve propagation and public participation to achieve high quality services of government websites

The service operation of government websites is crucial to the public's continuous use intention, and it directly influences their use perception and satisfaction. Therefore, government should promote its websites by various ways and lead the public to use the websites. For example, they can make full use of audios, videos, FLASH and other multimedia forms to enhance entertainment and attractiveness. Thus, government can improve the quality of systems and information of its websites to provide high quality public-oriented services.

(3) Establish a good service concept, emphasize on the process of service to achieve efficient service of government websites.

From interviews, we found government's ideas of website construction and service lagged behind. So, government is supposed to change the traditional ideas of handling affairs on site, develop informationalized ideas, pinpoint its functions and goals accurately, and build a good image of service oriented government. It is a problem that senior government officials only care about the input-output of government websites and ignore the service process. The process of service is what users can directly experience, so officials need to strengthen their understanding of the websites and attach importance to the service process. In addition, due to the lack of department cooperation, the information channels are narrow, and fail to form information networks. So government should enhance the integration of its websites, strengthen the cooperation between departments.

(4) Perfect the system construction, create a cultural atmosphere and construct guarantee mechanisms of government websites

The government ought to provide sufficient supports such as service mechanism, laws and regulations. Firstly, improve the information disclosure system to ensure the public' s right to know and trust perception. Secondly, perfect the security mechanism of government websites to improve the public's perception of safety. Thirdly, specify the responsibilities of service assurance, and implement the content protection mechanism. It is necessary to set up the accountability system, and establish content protection mechanisms to ensure the effectiveness of content maintenance. Fourthly, cultivate the information literacy of the public and build e-government culture to maintain the sustainable development.

Based on Grounded Theory, this paper explored the influential factors and mechanism of the public's continuous use intention of government websites and constructed a theoretical model. This paper also provided corresponding suggestions to current problems of government websites. However, there are limitations of this paper: (1) the design of interview questionnaire may lack precision because of the influence of the designers' knowledge and other factors; (2) the theory has certain limitations because the respondents' behaviors and modes of thinking were inevitably affected by factors such as region, social culture, and work. In order to improve the theory of this paper, we will carry out further empirical researches and applications of the theoretical model.

\section{ACKNOWLEDGMENTS}

First, we would like to thank the authors of the references, yearbooks and statistical reports; secondly, we would like to thank the students in the school, and it's the common communication and discusses that contribute to the birth of this paper.

\section{REFERENCES}

[1] West D M, Digital government: technology and public sector performance, Princeton University Press, 2005:46.

[2] Peters, Rob M., Janssen, Marijin, Van Engers and Tom M, Measurement of e-government impact: existing practices and shortcomings. ICEC '04 proceedings of the 6th international conference on electronic commerce, 2004, 60: 480-489.

[3] General Office of the State Council, Notification of $1^{\text {st }}$ Nationwide Investigation of Government Websites.(in Chinese) http://www.gov.cn/zhengce/content/2015-12/15/content_10421.htm

[4] China Internet Network Information Center, The $36^{\text {th }}$ Statistical Report of the Development of China Internet Network (in Chinese) http://www.cnnic.net.cn/hlwfzyj/hlwxzbg/hlwtjbg/201507/t20150722_5 2624.htm

[5] Research Group of the E-government Construction at the County Level in Fujian Province, Survey report on e-government construction of the counties in fujian province, E-Government, 2011, 09: 77-85 (in Chinese)

[6] Ma Liang, Factors influencing the use of government websites: investigation of big cities in china, E-Government, 2014, 04: 34-48 (in Chinese)

[7] Fishbein $M$ and Ajzen I, Belief, attitude, intention and behavior: an introduction to theory and research, New Jersey: Addison-Wesley Reading, 1975:135.

[8] Guopeng Yin and Ling zhu, Habit: how does it develop, and affect continued usage of chinese users on social networking websites? [J]. Journal of Organizational and End User Computing, 2014, 26(4):1-22.

[9] Deliang Wang, Lingling Xu and Hock Chuan Chan, Understanding the continuance use of social network sites: a computer self-efficacy perspective, Behaviour \& Information Technology, 2015, 34(2):204-216.

[10] Wilson, H.S. and Hutchinson, S. A., Triangulation of qualitative methods: heideggerian hermeneutics and grounded theory, Qualitative Health Research, 1991,1(2), 263-276.

[11] N. R. Pandit, The creation of theory: a recent application of the grounded theory method, The Qualitative Report, 1996, 2 (4):1-20.

[12] Anselm Strauss and Juliet Corbin, Basics of qualitative research: techniques and procedures for developing grounded theory, Translated by Wu Zhiyi, Liao Meihua.Taipei: Tao Shi Culture Business Co., Ltd,2009:150.

[13] Fang Wang, Explaining the low utilization of government websites: using a grounded theory approach. Government Information Quarterly, 2014, 31:610-621. 